\title{
Extinction of Conservation and Transitivity of Weight ${ }^{1}$
}

\author{
Scott A. Miller and Leann Lipps \\ University of Michigan
}

\begin{abstract}
This study attempted to determine whether children will relinquish their belief in Piagetian concepts upon presentation of disconfirming evidence. Two age groups were tested: third- and fourth-grade, and sixth-grade. Conservation of weight and transitivity of weight were the concepts examined; the discrepant feedback consisted of three trials with either nonconservation or nontransitivity outcomes. Resistance or extinction was inforred from the subject's explanations for the outcomes, his responses on subsequent trials, and his performance on a one-month delayed posttest. Conservation subjects showed only moderate resistance by any of these measures; furthermore, there was no evidence of a developmental increase in resistance. Transitivity subjects, in contrast, were much less likely than conservers to change their judgments on either the initial or the delayed test; they also showed the expected developmental incrcase in resistance to extinction. The results are interpreted as indicating that Piagetian concepts may vary in the extent to which they entail feelings of logical nccessity.
\end{abstract}

In assessing concepts of conservation, Piaget and his coworkers have long used a technique of counter-suggestion (e.g., "But another little boy told me ..."). Their feeling is that a child who truly understands conservation will be able to resist such contradictory suggestions. As an extension of this approach, Smedslund (1961) devised an extinction procedure for conservation of weight: A surreptitious removal of material during the transformation resulted in unequal weights and apparent nonconservation. In this technique, therefore, the child was confronted not only with a counter-suggestion but with counter-evidence. Smedslund found that children who had been trained to conserve were unable to resist extinction, suggesting that the obtained conservation had not been genuine. He concluded that the extinclion procedure had served its diagnostic function.

${ }^{1}$ This study was supported by a Faculty Research Grant No. 315660 from the Horace H. Rackham School of Graduate Studies, University of Michigan. The authors are grateful to Jean Gascon for his help with the apparatus, and to Celia Brownell and John Wilson for their invaluable assistance in the testing of subjects. We also thank the principal, teachers, and children of Spencer Elementary School, Whitmore Lake, Michigan. Authors' address: Department of Psychology, University of Michigan, Ann Arbor, Michigan 48104. 
A second finding of the study, however, complicates this conclusion. Of a control group of 13 natural conservers, only six were able to resist extinction. Subsequent studies of extinction in natural conservers have confirmed this low percentage, both for conservation of weight (Hall \& Kingsley, 1968; Hall \& Simpson, 1968; Kingsley \& Hall, 1967; Smith, 1968) and for conservation of liquid quantity (Brison, 1966; Sullivan, 1967). Most, in fact, have found even less evidence of resistance than that reported by Smedslund (see Miller, 1971, for a review of these studies). Such a result necessarily shifts the focus of such research from a concern with diagnosis to a concern with the nature of the conservation concept itself. For how can this ready abandonment of conservation be reconciled with the Piagetian claim that concepts of conservation are experienced as logically necessary (e.g., Piaget, 1971)?

One possible answer is to suggest that the extinction in these studies was apparent rather than real. There is almost certainly some truth to this suggestion. All of the studies have relied heavily on the child's immediate verbal response in inferring resistance or extinction (thus, e.g., "You took some clay away" would be a resistance response). Two (Hall \& Simpson, 1968; and Smith, 1968) have also examined judgments on subsequent conservation trials. In either case, to be credited with resistance a child has had to contradict what the experimenter has just demonstrated as true. Given the social pressure of the situation, some percentage of misdiagnosis seems assured.

Miller (1973) attempted to increase the probability that a continuing belief in conservation, if present, could be identified. Conservation of weight was the concept, with the discrepant feedback provided by means of a rigged balance. Each of the three feedback trials was followed by extensive probing concerning what had happened; in addition, there was a series of posttest trials without feedback, as well as further questioning at the end of the session. Surprise was measured as well as cognitive change. As expected, these procedures yielded more evidence of resistance than had been found in most previous studies. In particular, slightly more than half of the subjects were able to provide at least one resistance explanation (i.e., an explanation which denied the validity of the nonconservation outcome). On the other hand, there were still many children who appeared to abandon their belief in conservation upon presentation of one or two discrepant outcomes. In addition, measurable surprise was extremely rare.

The present study is an extension of Miller (1973). The central concern remains the problem of logical necessity-do feelings of necessity accompany an understanding of Piagetian concepts, and if so can their presence be inferred from the child's reactions when those concepts are violated? 
Three extensions of the previous study are included. The most important extension is the inclusion of an additional concept. As noted, extinction studies have focused on conservation, especially conservation of weight. The present study stays within the domain of weight but includes a separate condition for transitivity. In Piaget's theory, both conservation and transitivity are based in logical structures, and the same necessity is claimed for both. Any concept, however, no matter how logical at base, requires some grounding in physical experience and physical knowledge as well. It can be argued that the role of physical experience is in general greater for conservation than it is for transitivity. For conservation, the child must learn to cope with the effects of numerous different transformations in numerous content domains-transformations, moreover, which have varying effects depending on the domain in question (a particular transformation, e.g., may conserve weight but not length). For transitivity, there are no transformations, and the same abstract rules are directly applicable whatever the properties of the objects. Thus, however equivalent the underlying logical systems for the two concepts may be, their psychological application may differ. Application of principles of conservation may always be more contingent on-and hence more susceptible to disruption by-physical knowledge and physical feedback. The expectation from this analysis is that conservation will be more susceptible to extinction than will transitivity.

The second change from Miller (1973) is the addition of a delayed posttest. The rationale for such a test is obvious: If experimentally induced cognitive change is genuine, it should persist beyond the immediate testing situation. Only one extinction study (Hall \& Simpson, 1968) has included such a follow-up. Hall and Simpson did not fully analyze the results of their posttest; it appears from their tabular presentation, however, that a substantial number of original conservers did revert to conservation after a two-week interval.

The final change is an increase in the average age of the subjects. This increase stems from recent suggestions (e.g., Flavell, 1971) that the development of conservation may be a more extended process than has typically been thought. Perhaps feelings of logical necessity emerge only some time after the initial appearance of the concept. The current study retains two age levels as in Miller (1973) ; each group, however, is a year older than in the previous study.

\section{METHOD}

\section{Subjects}

The subjects were $\mathbf{9 7}$ third-, fourth-, and sixth-grade children from a small town in southeastern Michigan. Of the 97 children, 68 were retained 
for the complete study. These 68 children were divided equally among four groups: a third- and fourth-grade conservation group (mean age $=$ 9-6), a third- and fourth-grade transitivity group (mean age $=9-2$ ), a sixth-grade conservation group (mean age $=11-9$ ), and a sixth-grade transitivity group (mean age $=11-8$ ). There were 9 boys and 8 girls in each group, with the exception of the sixth-grade conservation group, which contained 8 boys and 9 girls. The remaining 29 subjects were rejected because of failure to pass the pretest. There were 16 failures on the conservation task ( 8 at the younger and 8 at the older age) and 13 failures on the transitivity task (11 at the younger and 2 at the older age).

\section{Apparatus}

All testing was conducted in one room of a mobile laboratory trailer. The second room housed an unseen observer who recorded all relevant verbalizations.

The major piece of apparatus was a hanging-pan balance scale. Outcomes on the scale could be read in two ways: by the alignment or nonalignment of the two pans, or by the movement of a pointer attached to the scale arm. The scale was capable of providing accurate feedback as to relative weight; it was also capable of providing systematically distorted feedback. Embedded in its base were two electromagnets, between which hung a soft iron bar attached to the underside of the scale arm. Activation of either magnet attracted the bar, causing the arm to swing to one or the other side and creating the appearance of unequal weights. The magnets were activated by the depression of silent foot pedals concealed bencath the table.

\section{Procedure}

Pretest phase. The session began with some brief pretraining designed to assure that the subject understood how the scale operated. The pretest followed. Subjects were randomly assigned to either the conservation or the transitivity condition, within the constraint that the eventual group sizes be equal.

Each pretest contained five items. In the conservation test, three of the items were standard conservation-of-equality problems employing clay balls: two balls were weighed and found equal; one was then transformed and the conservation question asked. The transformations were into a pancake, a sausage, or three small balls. A fourth trial was a conservation-of-inequality: a ball was shown (by means of the foot pedal) to weigh more than a snake; the ball was then rolled into an identical snake and the conservation question asked. The fifth trial involved a different material: two cardboard squares were weighed and 


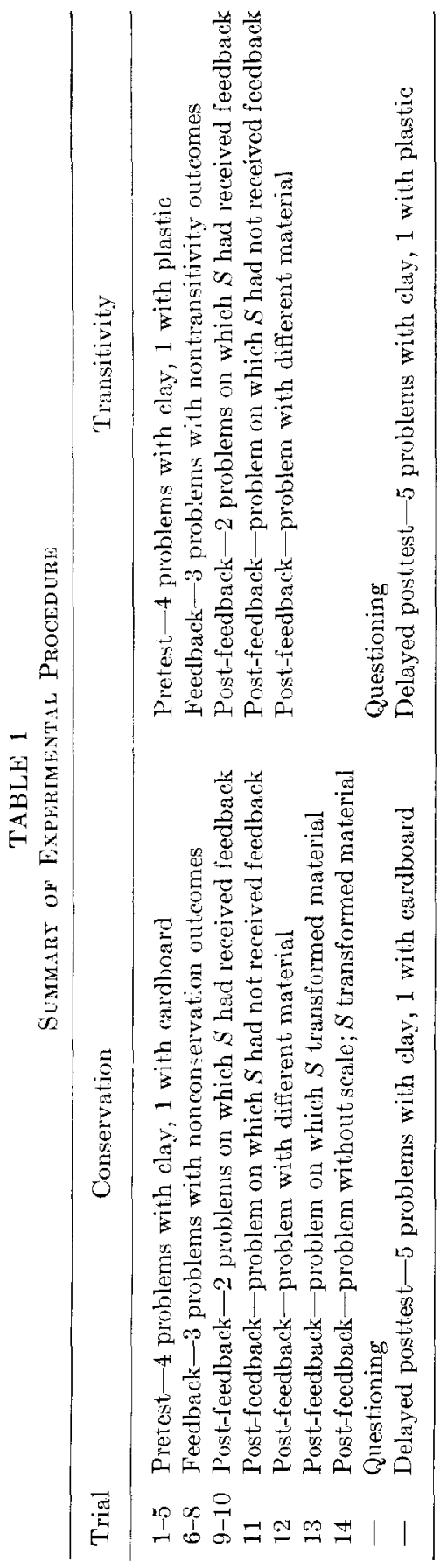


found equal; one was then folded twice and the conservation question asked. The order of the trials was balanced, the only constraints being that the snake trial not appear first and that the cardboard trial always appear last. The general form of the conservation question was, "Does the red weigh more, do they still weigh the same, or does the green weigh more?" followed by "Why do you think so?"

The transitivity problems also required the subject to judge the relative weights of two objects. In this casc, however, each of the objects was first weighed with a third, comparison object (a small piece of metal). The initial weighings were introduced as "a way to figure out what the two balls weigh." The subject was required to verbalize the results of the two weighings, as well as to recall them immediately before making his judgment. If he did not recall the results correctly, the weighings were repeated. The form of the transitivity question was, "Does the red weigh more, do they weigh the same, or does the green weigh more?" followed by "Why do you think so?"

On each trial, the size of the stimuli was opposed to the logical solution of the problem. The first four trials employed small (ca. 2-in. diameter) and large (ca. 31/2-in. diameter) clay balls. All of the balls weighed the same as the metal; apparent differences were produced by means of the foot pedal. The four trials (balanced for order within age groups) were as follows: small ball greater than metal, large ball less than metal; small ball greater than metal, large ball equal to metal; small ball equal to metal, large ball equal to metal; one small ball greater than metal, another small ball less than metal. The fifth trial involved a large and a small plastic barrel: the small barrel weighed more than the metal; the large barrel weighed less.

In both conditions, a child was retained for the complete study only if he answered all five problems correctly.

Feedback phase. The feedback phase followed immediately after the pretest. There were three feedback trials, each identical to one of the first four pretest trials. The various problems from the pretest appeared equally often in the fccdback phase, with the following exception: The snake trial was always one of the feedback trials for conservation, and the problem with two small balls was always one of the trials for transitivity (these trials permitted one case in which the outcome of the feedback was that the objects weighed the same).

Feedback was given following the subject's judgment and explanation. This feedback consistently disconfirmed either conservation or transitivity. On the conservation trials, the ball was made to appear heavier than the transformed object; the two snakes were made to appear equal. On the transitivity trials, the feedback was in the direction of the per- 
ceptual appearance. Thus, larger balls weighed more than smaller ones; two equal-sized balls weighed the same.

The experimenter waited for approximately three seconds after the onset of feedback, then asked "What happened?" There followed a period of sympathetic, flexible questioning designed to elicit the subject's true feelings about the outcome. Restatements of his prediction and the actual outcome were frequent, as were phrases such as "What do you really think?" and "Is there any other reason you can think of?" This questioning continued until the subject either appeared satisfied with an answer or was clearly unable to provide anything further.

Post-feedback phase. The post-feedback phase began with an assurance that the objects would no longer be weighed after the subject's judgment. The trials that followed were an attempt to depart gradually from the pressurcs of the fcedback situation. Each required both a judgment and an explanation for the judgment.

The conservation condition included six trials. The first two were repetitions (with different clay balls) of the two conservation-of-equality problems on which the subject had received feedback. The third involved the conservation-of-equality problem from the pretest which had not been included in the feedback phase. The fourth was a repetition of the trial with cardboard squares from the pretest.

The final two trials were additional repetitions of the first two conservation-of-equality problems; in this case, however, the subject rather than the experimenter transformed the material. In addition, the final trial was performed without use of the scale. The subject held the two balls to determine their equality, transformed one, gave his conservation judgment and explanation, then held the slimuli again and judged their relative weights. Conservation was credited only if the child gave an equality response both before and after holding the stimuli.

The post-feedback phase for transitivity included four trials. The pattern was similar to that for conservation: two problems on which the subject had received feedback, followed by a third problem from the pretest on which feedback had not been given, followed by the problem with different material.

For all subjects, the session concluded with a period of questioning which sought to elicit the child's true reactions to what had happened. The probing was similar to that on the feedback trials. Typically, it included verbal formulations of the problem, challenges to the child's answers (e.g., "But when we started you told me that...."), and requests to seek for explanations. The experimenter concluded by asking the child not to discuss the experiment with any of the other children.

Delayed posttest. The subjects were brought back to the trailer approximately one month after the first session. Not all children could be 
retested; the final $N$ s were 15 for the young conservers, 14 for the old conservers, 16 for the young transitivity group and 14 for the old transitivity group. The setting, stimuli, and scale were identical to those of the first session. The experimenter was different - a second adult female, not seen by the child previously.

The posttest contained six trials. The first five trials were identical to the pretest. The sixth trial was a repetition of the first, but with feedback provided following the subject's judgment and explanation. The feedback in this case was honest-i.e., either a conservation or a transitivity outcome. The child's prediction was elicited prior to the feedback and his explanation following it. The experimenter then demonstrated the trick and explained to the child that his initial answer had been correct. The child was again requested not to discuss the study with other children.

\section{Scoring}

The explanations which accompanied conservation and transitivity judgments were rated for logical adequacy. A conservation explanation was considered adequate if it fell into one of the standard Genevan categories (inversion, compensation, etc.). An explanation for transitivity was considered adequate if it referred accurately to both of the initial premises. Inter-rater reliabilities were $96 \%$ for conservation explanations and $98 \%$ for transitivity answers.

The explanations advanced for nonconservation or nontransitivity (either in response to feedback or because the subject had changed his prediction) were categorized into types; these types are presented in Table 2. Inter-rater agreement was $90 \%$ for nonconservation explanations and $87 \%$ for nontransitivity explanations. In cases of disagreement, the two raters discussed the explanation and arrived at a common judgment.

\section{RESULTS}

\section{Pretest}

Three findings from the pretest should be briefly noted. First, $95 \%$ of the conservation answers and $95 \%$ of the transitivity answers were accompanied by adequate explanations. Second, there were no significant differences at either age in the relative difficulty of the two tasks. Third, there were no sex differences on either task at either age.

\section{Immediate Test}

There were eight conservation trials and six transitivity trials following the first occurrence of feedback. The proportion of trials with changed 


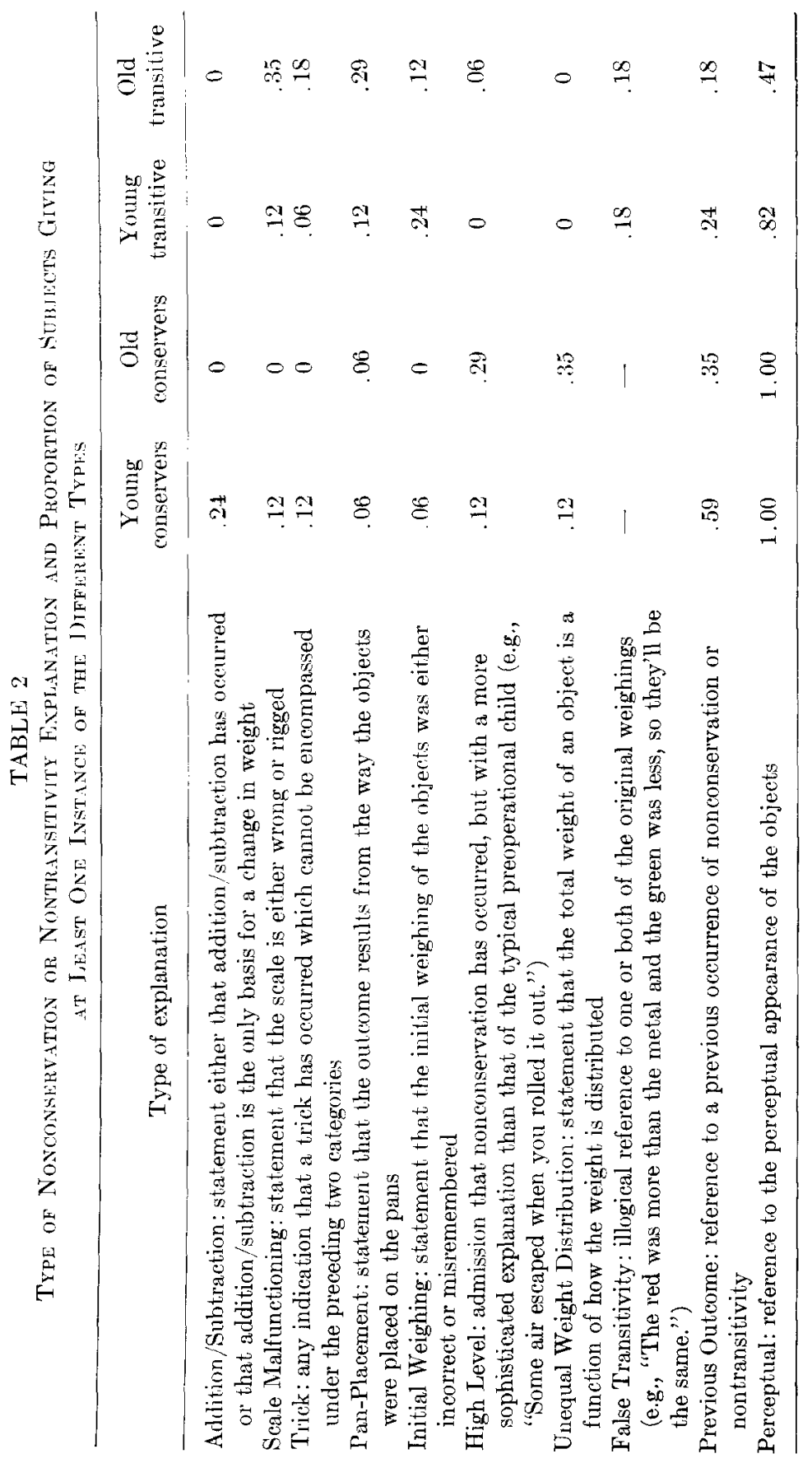


judgments (i.e., either nonconservation or nontransitivity judgments) is indicated in Table 3. A two-way (condition by age) analysis of variance revealed a significant main effect of condition $(F(1,64)=48.39, p<.01)$ and a significant condition by age interaction $(F(1,64)=9.51, p<.01)$. The main effect of age was not significant $(F(\mathbf{1 , 6 4 )}<1)$.

The source of the significant interaction is evident from the means. Transitivity subjects showed the expected decrease in changed judgments with increasing age; for conservers, however, there was a (nonsignificant) increase. Another way to state this finding is to note that the difference between conservation and transitivity, although present at both ages, was much greater for the older subjects.

Figure 1 indicates the percentage of changed judgments as a function of trial. In general, the pattern seems similar to what would be expected from the nature of the trials, although the last two trials in the conservation condition did prove unexpectedly difficult. Given the difficulty of these trials, it should be noted that all of the preceding between-group comparisons remain significant if only the first six conservation trials (those most directly comparable to the transitivity trials) are analyzed.

An analysis of incorrect answers must be supplemented by a consideration of the explanations which accompany the answers. Of particular interest is the proportion of wrong answers which are accompanied by Perceptual explanations only (this being the predominant explanation given by children who have not yet acquired the concept). As Table 3 indicates, imposition of such a criterion reduces the degree of apparent change for all groups. Analysis of variance again revealed a significant effect of condition $(F(1,64)=25.35, p<.01)$ and a significant condition

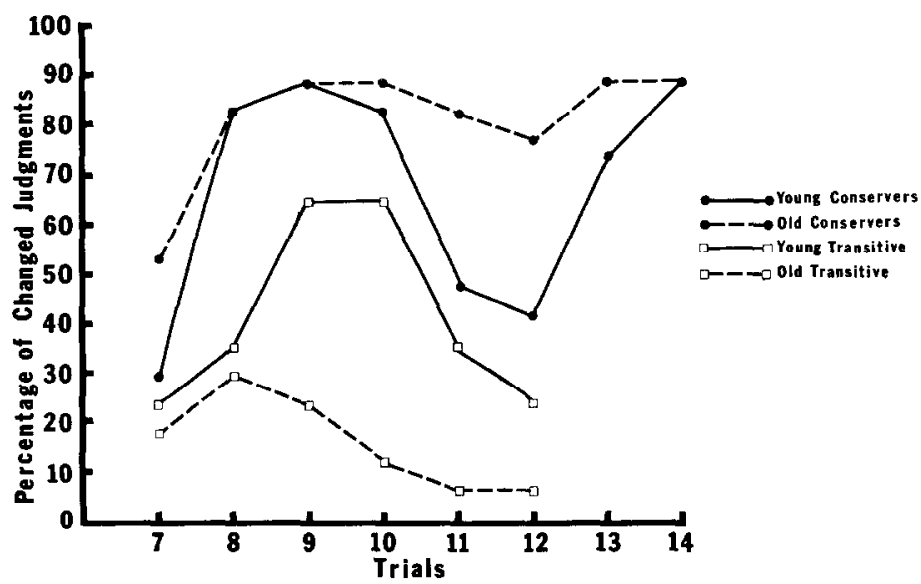

FIG. 1. Change in judgments by the four groups across trials. 


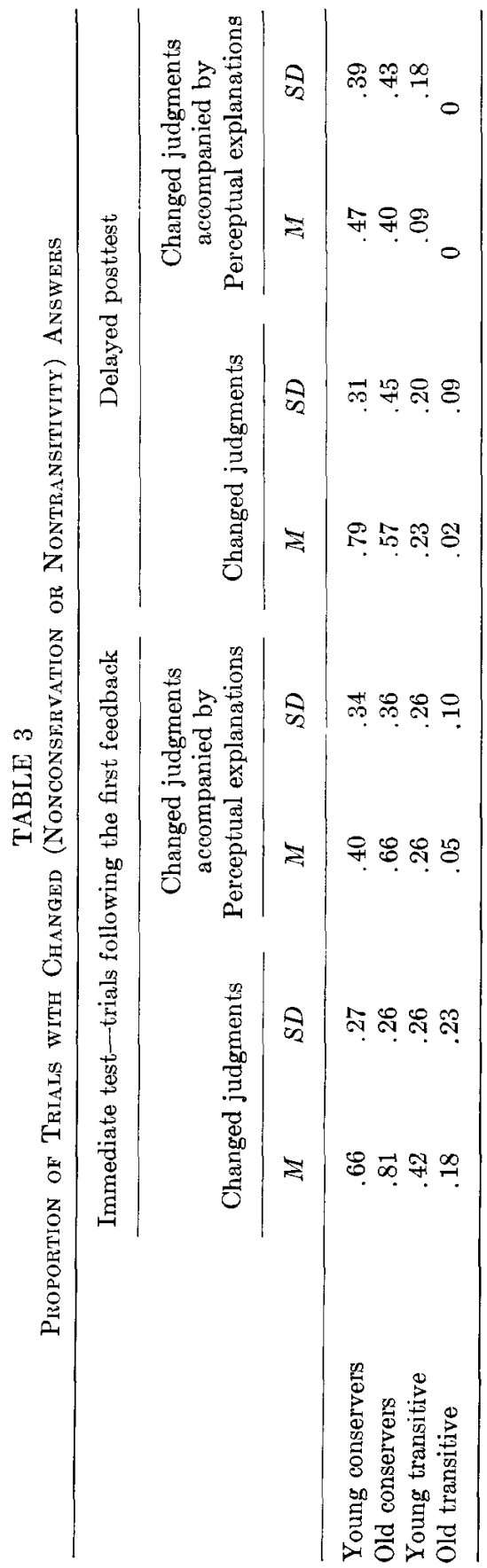


by age interaction $(F(1,64)=8.60, p<.01)$; the effect of age was again not significant $(F(1,64)<1)$. As was the case for changed judgments alone, the between-group comparisons remain significant if only the first six conservation trials are analyzed. They also remain significant if the "typical" nontransitivity explanation is broadened to include False Transitivity as well as Perceptual. (That the eategory should be expanded in this way is suggested by the fact that $26 \%$ of the incorrect pretest answers were accompanied by False 'Iransitivity explanations.) 'The broadened criterion increases the proportion of trials with changed answers to .31 for the younger transitivity group and .11 for the older.

The analyses thus far have focused on the average degree of change shown by the various groups. It is also possible to examine individual patterns of response. Of the 34 conservers, all but one (a subject from the younger group) gave at least one nonconservation judgment on trials 7 through 12 . In contrast, 11 transitivity subjects (two younger and nine older) never gave a nontransitivity judgment. If the criterion of a Perceptual or False Transitivity explanation is added, the number of subjects who never change is 4 for the young conservers, 3 for the old conservers, 3 for the young transitivity group, and 12 for the old transitivity group.

Explanations which did not fall into the Perceptual or False Transitivity categories provide the other major basis for analysis. The relevant data appeared in Table 2. The proportions in the table are based on explanations for outcomes on the feedback trials, explanations in support of nonconservation or nontransitivity judgments on trials 7 through 14 , and responses to the questioning at the end of the session.

The clearest indications of resistance are provided by explanations from the first five categories, each of which denies that the unexpected outcome has really occurred. The proportion of subjects offering at least one such response was $47 \%$ for the young conservers, $6 \%$ for the old conservers, $35 \%$ for the young transitivity group, and $59 \%$ for the old transitivity group. The relevant between-group comparisons yielded two significant results: Young conservers offered more resistance responses than old conservers ( $p=.008$, Fisher's Exact Test), and the old transitivity group offered more such explanations than did old conservers $(p=.001$, Fisher's Exact Test).

In the study by Miller (1973), the categories of High Level and Unequal Weight Distribution were utilized significantly more often by old conservers than by young conservers. It was argued there that such responses reflect a "sophisticatcd" form of nonconscrvation: an admission that weight has changed, but with an explanation virtually never given by the typical nonconserver. The present study produced a similar de- 
velopmental trend among the conservers; the differences, however, were of only borderline significance ( $p=.07$, Fisher's Exact Test).

One sort of response not captured in the table is failure to provide any explanation at all for the unexpected outcomes on the feedback trials. It could be argued that such failures reflect at least a minimal form of resistance, given the extensive probing and the obvious perceptual difference between the stimuli. Failures to respond were much more common for transitivity subjects, occurring 40 times across the three feedback trials, as compared to 14 instances for conservers.

\section{Delayed Posttest}

The proportion of posttest trials with changed answers is indicated in Table 3. Among transitivity subjects the posttest produced the expected reversion toward the earlier level of functioning. The performance of the older subjects, in fact, was virtually identical to their original pretest performance (adding False Transitivity explanations to Perceptual increases the proportion of trials with changed judgments accompanied by "typical" explanations to .17 and .01 for the younger and older transitivity groups respectively). The pattern for conservers was more complicated. While the older subjects did show a moderate improvement after the delay, the younger subjects actually gave more nonconservation answers than on the immediate test. For both groups, the degree of apparent nonconservation remained substantial.

\section{DISCUSSION}

The results for conservation proved quite similar to those of past research. The degrec of apparent resistance was moderate; if anything, it was slightly lower than in Miller (1973). There was little evidence of increased resistance with increasing age; on the immediate test, in fact, the differences were in favor of the younger subjects. There was also little indication of a return to conservation judgments on the delayed posttest.

The picture for transitivity was quite different. Transitivity subjects were less likely than conservers to offer changed judgments, and less likely to accompany changed judgments that did occur with appropriate explanations. Unlike conservers, transitivity subjects showed the expected decrease in changed predictions with increasing age, as well as the expected improvement following the one-month delay. The degree of apparent extinction after one month was low at the younger age and zero at the older.

Changed judgments provide one basis for inferring change; the nature of the subject's explanations provides the other. The contrast between 
conservation and transitivity subjects was less marked on the latter measure. A clear difference emerged at the older age in the direction of greater resistance by the transitivity subjects; there were no differences, however, at the younger age. It should be noted, of course, that such comparisons must be affected to some extent by the fact that the conservation and transitivity conditions were not equivalent in their range of possible explanations. In particular, the categories of Addition/Subtraction, Unequal Weight Distribution, and High Level were undoubtedly more available for conservers, whereas those of Initial Weighing and (by definition) False Transitivity were more available for transitivity subjects.

The question of the comparability of explanations raises the more general question of the comparability of the two extinction manipulations. Any study which compares two concepts must attempt to make its tests as comparable as possible; otherwise differences between the concepts may simply reflect different "hit rates" of the tests (Flavell, 1971). The extinction procedures utilized here would appear to meet this requirement: Both involved the same materials and the same scale; both included three trials, presented in the same general way; and in both there was a clear and consistent pcrecptually-based wrong answer. It seems quite likely, therefore, that differences in extinction really do reflect the differential certainty with which the two concepts are held.

As noted, the findings for conservation confirm those of previous studies. They suggest that a sizable proportion of conservers who have mastered conservation of weight by the standard measures do not experience that concept as logically necessary. It is true, of course, that the possibility of misdiagnosis remains; one direction for future research, therefore, would be to incorporate still further methodological controls for such "pseudoextinction." Another direction would be to develop a variety of pretest measures in an attempt to distinguish between "conservers" (by the usual criteria) who can resist extinction and those who cannot. Finally, the possibility of developmental changes beyond the grade-school age remains; there may still be a point, perhaps only in adulthood, when virtually all subjects would resist. The evidence for college-aged populations is thus far contradictory, Hall and Kingsley (1968) reporting little evidence of resistance, Miller, Schwartz, and Stewart (1973) finding very strong resistance.

The present results give little indication that transitivity can be extinguished. This concept really does seem to possess the underlying necessity of which Piaget has written. As suggested earlier, the difference between conservation and transitivity may lie in the extent to which the relevant logical structures must be supplemented by "real-world" knowl- 
edge concerning the properties of objects. Transitivity requires little such supplementation, and is correspondingly little affected when physical experience is put in opposition to logical structuring. Conservation requires more supplementation, and the disrupting effects of discrepant feedback are correspondingly greater. By this analysis, the two concepts may be equivalent in abstract logical structure; they differ, however, in the degree to which this logical structuring is expressed in psychological feelings of necessity.

\section{REFERENCES}

Bnison, D. W. Aeccleration of conservation of substance. Journal of Genetic Psychology, 1966, 109, 311-322.

Flavell, J. H. Stage-related properties of cognitive development. Cognitive Psychology, 1971, 2, 421-453.

HaLl, V. C., \& Kingseer, R. Conservation and equilibration theory. Joumal of Genetic Psychology, 1968, 113, 195-213.

HaLl, V. C., \& Simpson, G. J. Factors influencing extinction of weight conservation. Merrill-Palmer Quarterly, 1968, 14, 197-210.

Kingsley, R., \& HatL, V. C. Training conservation of weight and length through learning sets. Child Development, 1967, 38, 1111-1126.

Miller, S. A. Extinction of conservation: A methodological and theoretical analysis. Merrill-Palmer Quarterly, 1971, 17, 319-334.

Mrller, S. A. Contradiction, surprise, and cognitive change: The effects of disconfirmation of belief on conservers and nonconservers. Journal of Experimental Child Psychology, 1973, 15, 47-62.

Miller, S. A., Schwartz, L. C., \& Stewart, C. An attempt to extinguish conservation of weight in college students. Developmental Psychology, 1973, 8, 316.

Piaget, J. Biology and knowledge. Chicago: University of Chicago Press, 1971.

Smedsutnd, J. The acquisition of conservation of substance and weight in children. III. Extinction of conservation of weight acquired "normally" and by means of empirical controls on a balance scale. Scandinavian Journal of Psychology, 1961, 2, 85-87.

Sмrth, I. D. The effects of training procedures upon the acquisition of conservation of weight. Child Development, 1968, 39, 515-526.

Sullrvan, E. V. Acquisition of conservation of substance through film modeling techniques. In D. W. Brison \& E. V. Sullivan (Eds.), Recent research on the acquisition of conservation of substance. Ontario Institute for Studies in Education, 1967. Pp. 11-23. 